\title{
Korovkin and Weierstrass Approximation via Lacunary Statistical Sequences
}

\author{
${ }^{1}$ Richard F. Patterson and ${ }^{2}$ Ekrem Savaş \\ ${ }^{1}$ Department of Mathematics and Statistics, \\ University of North Florida Jacksonville, Florida, 32224, USA \\ ${ }^{2}$ Department of Mathematics, Faculty of Yüzüncü Yil University Education, Van, Turkey
}

\begin{abstract}
In this study we shall extended Korovkin and Weierstrass approximation theorem to lacunary statistical convergent sequences. In addition, to these approximation theorems, we established also introduced lacunary statistically convergent of degree $\beta$ and establish a corresponding Korovkin type theorem namely the following:

If the sequence of positive linear operators $\mathrm{P}_{n}: \mathrm{C}_{M}[\mathrm{a}, \mathrm{b}] \rightarrow \mathrm{B}[\mathrm{a}, \mathrm{b}]$ satisfies the conditions:

$* \quad\left\|\mathrm{P}_{n}(1, \mathrm{x})-1\right\|_{\beta} \rightarrow 0\left(\mathrm{~S}_{\theta}^{\beta_{1}}\right)$ as $\mathrm{r} \rightarrow \infty$,

$* \quad\left\|\mathrm{P}_{n}(\mathrm{t}, \mathrm{x})-\mathrm{x}\right\|_{B} \rightarrow 0\left(\mathrm{~S}_{\theta}^{\beta_{2}}\right)$ as $\mathrm{r} \rightarrow \infty$ and

$* \quad\left\|\mathrm{P}_{n}\left(\mathrm{t}^{2}, \mathrm{x}\right)-\mathrm{x}^{2}\right\|_{B} \rightarrow 0\left(\mathrm{~S}_{\theta}^{\beta_{3}}\right)$ as $\mathrm{r} \rightarrow \infty$, then for any function $\mathrm{f} \in \mathrm{C}_{M}[\mathrm{a}, \mathrm{b}]$, we have $\| \mathrm{P}_{n}(\mathrm{f}, \mathrm{x})-$ $(\mathrm{x}) \|_{B} \rightarrow 0\left(\mathrm{~S}_{\theta}^{\beta}\right)$ as $\mathrm{r} \rightarrow \infty$ and $\beta=\min \left\{\beta_{1}, \beta_{2}, \beta_{3}\right\}$.
\end{abstract}

Key words: Double Lacunary Sequence, P-Convergent

\section{INTRODUCTION}

The concept of statistical convergence was introduced by Fast in 1951. A complex number sequence $\mathrm{x}$ is said to be statistically convergent to the number $\mathrm{L}$ if for every $\varepsilon>0$

$$
\operatorname{Lim}_{n} \frac{1}{n}\{\mathrm{k}<\mathrm{n}: \mid \mathrm{x}-\mathrm{L} \mathrm{l} \geq \varepsilon\} \mid=0
$$

where by $\mathrm{k} \leq \mathrm{n}$ we mean that $\mathrm{k}=0,1,2, \ldots, \mathrm{n}$ and the vertical bars indicate the number of elements in the enclosed set. In this case we write $\mathrm{st}_{1^{-}} \operatorname{Lim} \mathrm{x}=\mathrm{L}$ or $\mathrm{x}_{k} \rightarrow \mathrm{L}\left(\mathrm{st}_{1}\right) . \quad$ By a lacunary $\theta=\left(\mathrm{k}_{r}\right) ; \mathrm{r}=0,1,2, \ldots .$. where $\mathrm{k}_{0}=0$, we shall mean an increasing sequence of non-negative integers with $\mathrm{k}_{r^{-}} \mathrm{k}_{\mathrm{r}-1} \rightarrow \infty$ as $\mathrm{r} \rightarrow \infty$. The intervals determined by $\theta$ will be denoted by $\mathrm{I}_{r}=\left(\mathrm{k}_{r-1}, \mathrm{k}_{r}\right]$ and $\mathrm{h}_{r}=\mathrm{k}_{r}-\mathrm{k}_{r-1}$. The ratio $k_{r} / k_{r-1}$ will be denoted by $\mathrm{q}_{r}$.

The following concept was presented by Fridy and Orhan. Let $\theta$ be a lacunary sequence; the number sequence $\mathrm{x}$ is $\mathrm{S}_{\theta^{-}}$convergent to $\mathrm{L}$ provided that for every $\mathcal{E}>0$

$$
\operatorname{Lim}_{r} \frac{1}{h_{r}}\left|\left\{\mathrm{k} \in I_{r}|\mathrm{x}-\mathrm{L}| \geq \varepsilon\right\}\right|=0
$$

in this case we write $\mathrm{S}_{\theta^{-}} \operatorname{Lim} \mathrm{x}=\mathrm{L}$ or $\mathrm{x}_{k \rightarrow} \mathrm{L}\left(\mathrm{S}_{\theta}\right)$. It is knownc that every convergaent sequence is statistical convergent, but not very statistical convergent sequence is convergent. The space of all functions that are continuous on $[\mathrm{a}, \mathrm{b}]$ and bounded by the number $\mathrm{M}$, shall be denoted by $\mathrm{C}_{M}[\mathrm{a}, \mathrm{b}]$. Also, $\mathrm{B}[\mathrm{a}, \mathrm{b}]$ is the space of all bounded function with norm $\|\mathrm{f}\|_{B}=\sup _{a \leq x \leq b}|\mathrm{f}(\mathrm{x})|$.
In 1960 Korovkin presented the following approximation theorem:

Theorem 2.1: If the sequence of positive linear operators $\mathrm{P}_{n}: \mathrm{C}_{M}[\mathrm{a}, \mathrm{b}] \rightarrow \mathrm{B}[\mathrm{a}, \mathrm{b}]$ satisfies the conditions:

* $\quad\left\|\mathrm{P}_{n}(1, \mathrm{x})-1\right\|_{B} \rightarrow 0$ as $\mathrm{n} \rightarrow \infty$,

* $\quad\left\|\mathrm{P}_{n}(\mathrm{t}, \mathrm{x})-\mathrm{x}\right\|_{B} \rightarrow 0$ as $\mathrm{n} \rightarrow \infty$ and

$* \quad\left\|\mathrm{P}_{n}\left(\mathrm{t}^{2}, \mathrm{x}\right)-\mathrm{x}^{2}\right\|_{B} \rightarrow 0$ as $\mathrm{n} \rightarrow \infty$,

then for any function $\mathrm{f} \in \mathrm{C}_{M}[\mathrm{a}, \mathrm{b}]$, we have

$$
\left\|\mathrm{P}_{n}(\mathrm{f}, \mathrm{x})-\mathrm{f}(\mathrm{x})\right\|_{B} \rightarrow 0 \text { as } \mathrm{n} \rightarrow \infty .
$$

This type of approximation was first introduced to statistical convergent by Gadjiev and Orhan in 2002. This introduction was accomplished by the presentation of a statistical analog of Korovkin theorem. In addition to this extension Gadjiev and Orhan observes that these theorem can be viewed as a Weierstrass approximation theorem via statistical convergent. The goal of this study is to extends Gadjiev and Orhan results to lacunary statistical convergent and observes that we have similar Weierstrass type approximation for lacunary statistical convergent sequences.

\section{RESULTS}

Theorem 2.1: If the sequence of positive linear operators $\mathrm{P}_{n}: \mathrm{C}_{M}[\mathrm{a}, \mathrm{b}] \rightarrow \mathrm{B}[\mathrm{a}, \mathrm{b}]$ satisfies the conditions: 
* $\quad\left\|\mathrm{P}_{n}(1, \mathrm{x})-1\right\|_{B} \rightarrow 0\left(\mathrm{~S}_{\theta}\right)$ as $\mathrm{r} \rightarrow \infty$,

$* \quad\left\|\mathrm{P}_{n}(\mathrm{t}, \mathrm{x})-\mathrm{x}\right\|_{B} \rightarrow 0\left(\mathrm{~S}_{\theta}\right)$ as $\mathrm{r} \rightarrow \infty$ and

$* \quad\left\|\mathrm{P}_{n}\left(\mathrm{t}^{2}, \mathrm{x}\right)-\mathrm{x}^{2}\right\|_{B} \rightarrow 0\left(\mathrm{~S}_{\theta}\right)$ as $\mathrm{r} \rightarrow \infty$

then for any function $\mathrm{f} \in \mathrm{C}_{M}[\mathrm{a}, \mathrm{b}]$, we have $\left\|\mathrm{P}_{n}(\mathrm{f}, \mathrm{x})-\mathrm{f}(\mathrm{x})\right\|_{B} \rightarrow 0\left(\mathrm{~S}_{\theta}\right)$ as $\mathrm{r} \rightarrow \infty$.

Proof: We shall follow the proof of Gadjiev and Orhan of [3] through the first stages for this proof. Since $f$ is bounded by a real number $\mathrm{M}$, we have the following

$$
|f(t)-f(x)| \leq 2 M
$$

for all $\mathrm{t}$ and $\mathrm{x}$. Also, since $\mathrm{f}$ is continuous on $[\mathrm{a}, \mathrm{b}]$ then for given $\varepsilon>0$

$$
|\mathrm{f}(\mathrm{t})-\mathrm{f}(\mathrm{x})|<\mathcal{E}
$$

for all $\mathrm{t}$ and $\mathrm{x}$ satisfying $|\mathrm{t}-\mathrm{x}|<\delta$. For $\mathrm{x} \in[\mathrm{a}, \mathrm{b}]$ let us consider $\mathrm{g}_{x}(\mathrm{t})=(\mathrm{t}-\mathrm{x})^{2}$, for $|\mathrm{t}-\mathrm{x}| \leq \delta$. This implies that $\left\|g_{x}(\mathrm{t})\right\| \leq \delta$ where $\delta$ is a fixed real number. Since $\mathrm{P}_{n}$ is a sequence of linear operators we obtain the following:

$\mathrm{P}_{n}(\mathrm{f}(\mathrm{t}), \mathrm{x})-\mathrm{f}(\mathrm{x})=\mathrm{P}_{n}(\mathrm{f}(\mathrm{t})-\mathrm{f}(\mathrm{x}), \mathrm{x})+\mathrm{f}(\mathrm{x})\left(\mathrm{P}_{n}(1, \mathrm{x}), \mathrm{x}\right)$.

As stated in [3] we are granted the following:

$$
\begin{aligned}
\left\|\mathrm{P}_{n}(\mathrm{f}(\mathrm{t}), \mathrm{x})-\mathrm{f}(\mathrm{x})\right\|_{B Z} & \leq\left(\varepsilon+M+\frac{2 M}{\delta^{2}}\right) \\
& \times\left\|\mathrm{P}_{n}(1, \mathrm{x})-1\right\|_{B} \\
& +\frac{4 M b}{\delta^{2}}\left\|\mathrm{P}_{n}(\mathrm{t}, \mathrm{x})-\mathrm{x}\right\|_{B} \\
& +\frac{2 M}{\delta^{2}}\left\|\mathrm{P}_{n}\left(\mathrm{t}^{2}, \mathrm{x}\right)-\mathrm{x}^{2}\right\|_{B} \\
& \leq \mathrm{K}_{1}\left(\left\|\mathrm{P}_{n}(1, \mathrm{x})-1\right\|_{B}\right. \\
& \left.+\left\|\mathrm{P}_{n}(\mathrm{t}, \mathrm{x})-\mathrm{x}\right\|_{B}\right) \\
& +\mathrm{K}_{1}\left\|\mathrm{P}_{n}\left(\mathrm{t}^{2}, \mathrm{x}\right)-\mathrm{x}^{2}\right\|_{B}
\end{aligned}
$$

where

$$
\mathrm{K}_{1}=\max \left\{\varepsilon+M+\frac{2 M}{\delta^{2}}, \frac{4 M b}{\delta^{2}}\right\}
$$

For $\varepsilon^{\prime}>0$ the last inequality implies the following:

$$
\begin{aligned}
\mid\{\mathrm{n} & \left.\in I_{r}:\left\|\mathrm{P}_{n}(\mathrm{f}, \mathrm{x})-\mathrm{f}(\mathrm{x})\right\|_{B} \geq \mathcal{E}^{\prime}\right\} \mid \\
\leq & \mid\left\{\left\|\mathrm{P}_{n}(1, \mathrm{x})-1\right\|_{B} \geq \frac{\mathcal{E}^{\prime}}{K_{1}}\right. \\
& +\left\|\mathrm{P}_{n}(\mathrm{t}, \mathrm{x})-\mathrm{x}\right\|_{B} \geq \frac{\mathcal{E}^{\prime}}{K_{1}} \\
& \left.+\left\|\mathrm{P}_{n}\left(\mathrm{t}^{2}, \mathrm{x}\right)-\mathrm{x}^{2}\right\|_{B} \geq \frac{\varepsilon^{\prime}}{K_{1}}\right\} \mid .
\end{aligned}
$$

Now let us consider the following sets

$$
\mathrm{D}=: \text { I }\left\{\mathrm{n} \in I_{r}: \| \mathrm{P}_{n}(1, \mathrm{x})-1\right)\left\|_{B}+\right\| \mathrm{P}_{n}(\mathrm{t}, \mathrm{x})-\mathrm{x} \|_{B}
$$

$$
\begin{gathered}
\left.+\left\|\mathrm{P}_{n}\left(\mathrm{t}^{2}, \mathrm{x}\right)-\mathrm{x}^{2}\right\|_{B} \geq \frac{\varepsilon^{\prime}}{K_{1}}\right\} \mid, \\
\left.\mathrm{D}_{1}=: \mid\left\{\mathrm{n} \in I_{r}: \| \mathrm{P}_{n}(1, \mathrm{x})-1\right) \|_{B} \geq \frac{\varepsilon^{\prime}}{3 K_{1}}\right\} \mid, \\
\left.\mathrm{D}_{2}=: \mid\left\{\mathrm{n} \in I_{r}: \| \mathrm{P}_{n}(\mathrm{t}, \mathrm{x})-\mathrm{x}\right) \|_{B} \geq \frac{\varepsilon}{3 K_{1}}\right\} \mid \text { and } \\
\left.\mathrm{D}_{3}=: \mid\left\{\mathrm{n} \in I_{r}: \| \mathrm{P}_{n}\left(\mathrm{t}^{2}, \mathrm{x}\right)-\mathrm{x}^{2}\right) \|{ }_{B} \geq \frac{\varepsilon}{3 K_{1}}\right\} \mid .
\end{gathered}
$$

It is also clear that $\mathrm{D} \subset \mathrm{D}_{1} \cup \mathrm{D}_{2} \cup \mathrm{D}_{3}$. This grants us the following inequality

$$
\begin{aligned}
\mid\left\{\mathrm{n} \in I_{r}\right. & \left.:\left\|\mathrm{P}_{n}(\mathrm{f}, \mathrm{x})-\mathrm{f}(\mathrm{x})\right\|_{B} \geq \mathcal{E}^{\prime}\right\} \mid \\
& \leq \mid\left\{\left\|\mathrm{P}_{n}(1, \mathrm{x})-1\right\|_{B} \geq \frac{\mathcal{E}^{\prime}}{3 K_{1}}\right. \\
& +\left\|\mathrm{P}_{n}(\mathrm{t}, \mathrm{x})-\mathrm{x}\right\|_{B} \geq \frac{\mathcal{E}^{\prime}}{3 K_{1}} \\
& \left.+\left\|\mathrm{P}_{n}\left(\mathrm{t}^{2}, \mathrm{x}\right)-\mathrm{x}^{2}\right\|_{B} \geq \frac{\varepsilon}{3 K_{1}}\right\} \mid
\end{aligned}
$$

Therefore (1), (2) and (3) implies $\| \mathrm{P}_{n}$ (f, $\left.\mathrm{x}\right)$ $\mathrm{f}(\mathrm{x}) \|_{\beta} \rightarrow 0\left(\mathrm{~S}_{\beta}\right)$ as $\mathrm{r} \rightarrow \infty$. This completes the proof.

Theorem 2.2: If the sequence of positive linear operators $\mathrm{P}_{n}: \mathrm{C}_{M}[\mathrm{a}, \mathrm{b}] \rightarrow \mathrm{B}[\mathrm{a}, \mathrm{b}]$ satisfies the conditions:

$* \quad\left\|\mathrm{P}_{n}(1, \mathrm{x})-1\right\|_{B} \rightarrow 0\left(\mathrm{~S}_{\theta}\right)$ as $\mathrm{r} \rightarrow \infty$,

* $\quad\left\|\mathrm{P}_{n}(\mathrm{t}, \mathrm{x})-\mathrm{x}\right\|_{B} \rightarrow 0\left(\mathrm{~S}_{\theta}\right)$ as $\mathrm{r} \rightarrow \infty$ and

$* \quad\left\|\mathrm{P}_{n}(1, \mathrm{x})-1\right\|_{B} \rightarrow 0$ as $\mathrm{n} \rightarrow \infty$,

then for any function $\mathrm{f} \in \mathrm{C}_{M}[\mathrm{a}, \mathrm{b}]$, we are granted the following:

$$
\lim _{N} \frac{1}{N} \sum_{\mathrm{n}=1}^{\mathrm{N}}\|\mathrm{P}(\mathrm{f}(\mathrm{t}), \mathrm{x})-\mathrm{f}(\mathrm{x})\|_{\mathrm{B}}=0
$$

Proof: Since $\left\|\mathrm{P}_{n}(1, \mathrm{x})-1\right\|_{\beta} \rightarrow 0$ as $\mathrm{n} \rightarrow \infty$, there exists $\mathrm{M}_{1}$ such that for all $\mathrm{n},\left\|\mathrm{P}_{n}(1, \mathrm{x})\right\|_{\beta} \leq \mathrm{M}_{1}$. Thus for any $\mathrm{f} \in \mathrm{C}_{M}[\mathrm{a}, \mathrm{b}]$ and $\mathrm{n}=1,2,3, \ldots$ we have the following:

$$
\begin{aligned}
\left\|\mathrm{P}_{n}(\mathrm{f}, \mathrm{x})-\mathrm{f}(\mathrm{x})\right\|_{B} & \leq\|\mathrm{f}\|_{C}\left\|\mathrm{P}_{n}(1, \mathrm{x})\right\|_{B} \\
& +\|\mathrm{ff}\|_{C} \mathrm{IM}\left(\mathrm{M}_{1}+1\right) .
\end{aligned}
$$

We also know that

$\mathrm{P}_{n}(1, \mathrm{x})-1 \|_{B} \rightarrow 0$ as $\mathrm{n} \rightarrow \infty$ implies

$\left\|\mathrm{P}_{n}(1, \mathrm{x})-1\right\|_{B} \rightarrow 0\left(\mathrm{~S}_{\theta}\right)$ as $\mathrm{r} \rightarrow \infty$.

Thus by Theorem $2.1\left\|\mathrm{P}_{n}(\mathrm{f}, \mathrm{x})-\mathrm{f}(\mathrm{x})\right\|_{B} \rightarrow 0\left(\mathrm{~S}_{\theta}\right)$ as $\mathrm{r} \rightarrow \infty$.

In [2] Theorem 4 assures us that if a sequence is bounded and is $\mathrm{S}_{\theta}$-convergent then it is $(\mathrm{C}, 1)$ summable to the same limit. Thus

$$
\underset{N}{\operatorname{Lim}} \frac{1}{N} \sum_{\mathrm{n}=1}^{\mathrm{N}}\|\mathrm{P}(\mathrm{f}(\mathrm{t}), \mathrm{x})-\mathrm{f}(\mathrm{x})\|_{\mathrm{B}}=0
$$


This completes the proof.

The Weierstrass approximation theorem state the following: If $\mathrm{f}$ is a continuous function on $[\mathrm{a}, \mathrm{b}]$ then there is a sequence of polynomials $\left(\mathrm{PL}_{n}\right)$ such that

$$
\underset{n}{\operatorname{Lim}}\|\mathrm{PL}-\mathrm{f}\|_{C[a, b]}=0
$$

In this case we have $\| P_{n}$ (f, $\left.x\right)-f(x) \|_{\beta} \rightarrow 0\left(S_{\theta}\right)$ as $\mathrm{n} \rightarrow \infty$. This can be interpreted as follows:

Theorem 2.3: If $\mathrm{f}$ is a continuous function on $[\mathrm{a}, \mathrm{b}]$ then there is a sequence of polynomials which are lacunary statistically convergent to $f$ on this interval but not uniformly convergent.

Definition 2.1: The number sequence $\mathrm{x}=\mathrm{x}_{k}$ is lacunary statistically convergent to the number $\mathrm{L}$ with degree $0<\beta<1$, if for each $\mathcal{E}>0$

$$
\operatorname{Lim}_{r} \frac{1}{\left(h_{r}\right)^{1-\beta}}|\{\mathrm{k} \mathrm{I}|\mathrm{x}-\mathrm{L}| \geq \varepsilon\}|=0
$$

which we shall denote by $\mathrm{x}_{k}-\mathrm{L} \rightarrow 0\left(\mathrm{~S}_{\theta}^{\beta}\right)$.

Theorem 2.4: If the sequence of positive linear operators $\mathrm{P}_{n}: \mathrm{C}_{M}[\mathrm{a}, \mathrm{b}] \rightarrow \mathrm{B}[\mathrm{a}, \mathrm{b}]$ satisfies the conditions:

$$
\begin{aligned}
& * \quad\left\|\mathrm{P}_{n}(1, \mathrm{x})-1\right\|_{B} \rightarrow 0\left(\mathrm{~S}_{\theta}^{\beta_{1}}\right) \text { as } \mathrm{r} \rightarrow \infty \\
& * \quad\left\|\mathrm{P}_{n}(\mathrm{t}, \mathrm{x})-\mathrm{x}\right\|_{B} \rightarrow 0\left(\mathrm{~S}_{\theta}^{\beta_{2}}\right) \text { as } \mathrm{r} \rightarrow \infty \text { and } \\
& * \quad\left\|\mathrm{P}_{n}\left(\mathrm{t}^{2}, \mathrm{x}\right)-\mathrm{x}^{2}\right\|_{B} \rightarrow 0\left(\mathrm{~S}_{\theta}^{\beta_{3}}\right) \text { as } \mathrm{r} \rightarrow \infty \\
& \text { then } \quad \text { for any function } \mathrm{f} \in \mathrm{C}_{M}[\mathrm{a}, \mathrm{b}], \text { we } \\
& \text { have }\left\|\mathrm{P}_{n}(\mathrm{f}, \mathrm{x})-\mathrm{f}(\mathrm{x})\right\|_{B} \rightarrow 0\left(\mathrm{~S}_{\theta}^{\beta}\right) \text { as } \mathrm{r} \rightarrow \infty \text { and } \\
& \beta=\min \left\{\beta_{1}, \beta_{2}, \beta_{3}\right\} .
\end{aligned}
$$

Proof: We are granted the following results as in Theorem 2.1:

$$
\begin{aligned}
& \frac{\left|\left\{n \in I_{r}:\left\|P_{n}(f(t), x)-f(x)\right\|_{B} \geq \varepsilon^{\prime}\right\}\right|}{\left(h_{r}\right)^{1-\beta}} \\
& \quad \frac{\left|\left\{n \in I_{r}:\left\|P_{n}(1, x)-1\right\|_{B} \geq \frac{\varepsilon^{\prime}}{3 K_{1}}\right\}\right|}{\left(h_{r}\right)^{1-\beta_{1}}} \frac{h_{r}^{1-\beta_{1}}}{h_{r}^{1-\beta}} \\
& +\frac{\left|\left\{n \in I_{r}:\left\|P_{n}(t, x)-x\right\|_{B} \geq \frac{\varepsilon^{\prime}}{3 K_{1}}\right\}\right|}{\left(h_{r}\right)^{1-\beta_{2}}} \frac{h_{r}^{1-\beta_{2}}}{h_{r}^{1-\beta}} \\
& +\frac{\left|\left\{n \in I_{r}:\left\|P_{n}(t, x)-x\right\|_{B} \geq \frac{\varepsilon^{\prime}}{3 K_{1}}\right\}\right|}{\left(h_{r}\right)^{1-\beta_{3}}} \frac{h_{r}^{1-\beta_{3}}}{h_{r}^{1-\beta}}
\end{aligned}
$$

This inequality implies the desired results. This completes the proof

Finally we conclude this study by stating a theorem which characterizes Korovkin type theorem for lacunary statistical convergent sequences. To accomplish this we consider positive linear operations $\mathrm{P}_{n}$ from $\mathrm{L}_{p}[\mathrm{a}, \mathrm{b}]$ to $\mathrm{L}_{p}[\mathrm{a}, \mathrm{b}]$. We omit the proof since it involves known arguments which are used in Theorem 7 of [3].

Theorem 1.2: If the sequence of positive linear operators $\mathrm{P}_{n}: \mathrm{L}_{p}[\mathrm{a}, \mathrm{b}] \rightarrow \mathrm{L}_{p}[\mathrm{a}, \mathrm{b}]$ such that $\left\{\left\|\mathrm{P}_{n}\right\|\right\}$ is uniformly bounded and satisfy the following conditions

$* \quad\left\|\mathrm{P}_{n}(1, \mathrm{x})-1\right\|_{L_{p}} \rightarrow 0\left(\mathrm{~S}_{\theta}\right)$ as $\mathrm{r} \rightarrow \infty$,
$* \quad\left\|\mathrm{P}_{n}(\mathrm{t}, \mathrm{x})-\mathrm{x}\right\|_{L_{p}} \rightarrow 0\left(\mathrm{~S}_{\theta}\right)$ as $\mathrm{r} \rightarrow \infty$ and
$* \quad\left\|\mathrm{P}_{n}\left(\mathrm{t}^{2}, \mathrm{x}\right)-\mathrm{x}^{2}\right\|_{L_{p}} \rightarrow 0\left(\mathrm{~S}_{\theta}\right)$ as $\mathrm{r} \rightarrow \infty$, then for any function $\mathrm{f} \in L_{p}[\mathrm{a}, \mathrm{b}]$, we are granted the following:

$$
\left\|\mathrm{P}_{n}(\mathrm{f}, \mathrm{x})-\mathrm{f}(\mathrm{x})\right\|_{L_{p}} \rightarrow 0\left(\mathrm{~S}_{\theta}\right) \text { as } \mathrm{r} \rightarrow \infty .
$$

\section{CONCLUSION}

The results in this study add new ideas to the underlining theory of approximation. This was accomplished by presenting a series of extensions to the Korovkin theorem using lacunary sequences. There are many possible directions for these ideas to go, not the least of which is by using general summability methods. In addition, other important approximation theorems can be extended to lacunary sequence spaces. The methods incorporated throughout this study are classical summability methods, however modern analysis method may also shine light on these ideas.

\section{ACKNOWLEDGEMENTS}

The authors wish to thank the referee for his constructive comments and suggestions.

\section{REFERENCES}

1. Fast, H., 1951. Sur la convergence statistique, Collog. Math., 2: 241-244.

2. Fridy, J.A. and C. Orhan, 1993. Lacunary Statistical Summability, J. Math. Anal. Appl., 173: 497-504.

3. Gadjiev, A.D. and C. Orhan, 2002. Some approximation Theorems via Statistical Convergent, Rocky Mountain J. Math., 32: 129-138.

4. Korovkin, P.P., 1960. Linear operators and the theory of approximation, Delhi, India. 